\title{
Aum Shinrikyô : secte et violence (Partie 1)
}

\section{Sylvaine Trinh}

\section{OpenEdition}

\section{Journals}

\section{Édition électronique}

URL : http://journals.openedition.org/conflits/718

DOI : $10.4000 /$ conflits. 718

ISSN : $1777-5345$

Éditeur :

CCLS - Centre d'études sur les conflits lilberté et sécurité, L'Harmattan

\section{Édition imprimée}

Date de publication : 15 octobre 1998

ISSN : 1157-996X

\section{Référence électronique}

Sylvaine Trinh, « Aum Shinrikyô : secte et violence (Partie 1) », Cultures \& Conflits [En ligne], 29-30 | automne-hiver 1998, mis en ligne le 16 mars 2006, consulté le 30 mars 2021. URL : http:// journals.openedition.org/conflits/718; DOI : https://doi.org/10.4000/conflits.718

Ce document a été généré automatiquement le 30 mars 2021.

Creative Commons License 


\title{
Aum Shinrikyô : secte et violence (Partie 1)
}

\author{
Sylvaine Trinh
}

Aum Shinrikyô : secte et violence ${ }^{1}$

Aum Shinrikyô est le seul mouvement religieux qui ait jamais au Japon conçu et perpétré une agression tournée vers l'extérieur de lui-même, un meurtre massif et sans discrimination, avec l'attentat au gaz sarin dans le métro de Tokyo, le 20 mars 1995. Comment, pourquoi ce mouvement religieux a-t-il ainsi sombré à un moment donné dans une violence inouïe ? Celle-ci est-elle produite par la société japonaise, et plus précisément par son système social? Aurait-elle pu surgir ailleurs? Est-ce que ce que nous apprend Aum Shinrikyô concerne la seule société japonaise ou, plus largement la modernité?

Après l'attentat du 20 mars 1995, deux hypothèses principales ont inégalement retenu l'attention au Japon. L'hypothèse " idéologique " est de suite avancée : Aum Shinrikyô a voulu déstabiliser l'Etat en éliminant le plus grand nombre de fonctionnaires possible. Il s'agirait alors d'un pur attentat terroriste, de nature exclusivement politique, perpétré dans un pays qui a une expérience relativement faible du phénomène, limité à de rares groupes extrémistes, de gauche ou de droite - quelques événements ont ainsi marqué la décennie qui vient de s'écouler, tous liés à des logiques internes ${ }^{2}$. La seconde hypothèse, " pathologique ", repose sur l'analyse de psychiatres qui, après avoir étudié le cas de plusieurs ex-disciples de la secte traités dans les hôpitaux, concluent à un type de folie collective empêchant les personnes atteintes de contrôler leurs actes. Cette pathologie serait due à la sur-utilisation de drogues et aux conditions de vie au sein de la communauté religieuse. La première hypothèse est de nature socio-politique, centrée sur un acteur politico-religieux qui s'engage dans un rapport social conflictuel contre l'Etat, rapport qui ne trouve son expression que dans la violence. La seconde est de nature psycho-sociologique, centrée sur l'idée d'un sujet aliéné par les drogues ou par son mode de vie et dont la violence, tournée vers l'extérieur, ne ferait qu'exprimer sa propre souffrance. La première de ces deux hypothèses conduit à la condamnation sans appel des responsables, tandis que la seconde suggère l'irresponsabilité des coupables. 


$$
\text { les deux précédentes, et selon laquelle l'enjeu de la violence est de trier ceux qui seront }
$$
sauvés au moment du Jugement dernier. périodes qui marquent la vie de la secte. Cette étude met en évidence les processus qui mènent Aum Shinrikyô à la violence, repère les moments où la secte s'engage dans des actions plus irréversibles qu'auparavant, et met en relation les conditions dans lesquelles interviennent ces changements de nature dans la violence produite.

8 A posteriori, de nombreux indices auraient dû orienter la police japonaise vers Aum Shinrikyô bien avant l'attentat du métro au gaz sarin. Tokyo, se plaignent de fumées blanches nocives qui s'échappent de bâtiments appartenant à Aum Shinrikyô. Les autorités n'interviennent pas : il s'agit de bâtiments appartenant à une organisation religieuse.

Surtout, à Matsumoto, le 27 juin 1994, des vapeurs de sarin sont dispersées sur un parking de supermarché dans un quartier résidentiel, faisant sept morts et intoxiquant deux cents personnes. Un camion modifié avait servi à répandre le gaz. La police ne parvient pas alors à identifier les auteurs de cette attaque et avance le nom d'un certain Kono qui avait donné l'alerte. Le malheureux détenait à son domicile des substances chimiques dont il se servait pour entretenir son jardin et avait travaillé pour une entreprise de produits chimiques. Les médias s'emparent du nom et en font aussitôt l'auteur du drame. Désigné à tort, Kono, dont la femme gravement intoxiquée demeure dans un état très grave, est par la suite largement soutenu par diverses associations. Début juin 1995, trois chaînes de télévision leur présentent publiquement leurs excuses pour l'avoir traité comme le principal suspect et avoir annoncé qu'il avait produit le gaz toxique par erreur en voulant obtenir un herbicide. Le quotidien local, auquel Kono avait d'abord demandé vingt millions de yens en dommages et intérêts avant de retirer sa plainte, publie un article innocentant clairement l'accusé. La police, de son côté, " regrette " d'avoir interrogé, c'est-à-dire soupçonné, l'innocent Kono.

Plus tard, la culpabilité de la secte Aum Shinrikyô sera clairement établie dans l'attentat de Matsumoto et il se dira qu'il ne s'agissait que d'un essai, préparant l'attentat de Tokyo. Les " défauts " de cette première expérience auraient permis à la secte d'affiner la qualité du gaz produit ainsi que la fiabilité des appareillages de dispersion: la diffusion du gaz, dans ce premier attentat, ne s'effectue pas comme prévu et les analyses des résidus de sarin trouvés à Matsumoto révéleront la qualité moindre du gaz utilisé, par rapport à celle du sarin de Tokyo.

En juillet 1994, plusieurs résidents de Kamikuishiki, village d'une population dispersée de mille sept cents habitants dans la préfecture de Yamanashi où la secte s'est installée, se plaignent d'irritation des yeux et du nez et souffrent de nausées : une partie d'entre 
eux doivent alors être évacués. Les malaises sont dus à l'inhalation de vapeurs toxiques que les autorités locales ne parviennent pas à identifier. En décembre 1994, la police de Kamikuishiki découvre du matériel qui pourrait servir à fabriquer du gaz. En janvier 1995, Aum Shinrikyô, non sans cynisme, dépose plainte contre une entreprise qui aurait répandu du sarin dans les bâtiments religieux de Kamikuishiki. Le 5 mars 1995, onze personnes sont hospitalisées à Yokohama après avoir respiré des vapeurs dans le train et souffrent de divers malaises. Et le 15 mars, cinq jours avant l'attentat de Tokyo, sont découverts à Kasumigaseki trois attachés-cases pour le moins suspects : ils contiennent un liquide non identifié, des ventilateurs automatiques et des piles.

15 A l'ouest de l'Australie, vingt-quatre moutons sont retrouvés morts et les analyses révèlent qu'ils ont inhalé un gaz qui pourrait provenir de la décomposition du sarin. Aum Shinrikyô avait acquis dans cette région un ranch en juin 1993 et y avait apporté des produits chimiques pour y prospecter de l'or. Et plusieurs aborigènes vivant près de la propriété rapportent qu'ils ont vu en août 1994 cinq hommes arpenter la propriété avec de longs costumes et des casques. La propriété fut revendue juste après, en septembre 1994.

L'enchaînement de ces faits précédant l'attentat de Tokyo mettait en cause fortement la secte. A cela s'ajoutaient de nombreuses plaintes déposées par les familles d'adeptes de Aum Shinrikyô, des mises en garde publiques de la part d'avocats et de diverses associations. La non-intervention de la police avant le 20 mars 1995 reste incomprise et largement inexplicable.

L'enlèvement de Kariya

18 L'affaire Kariya est sans doute déterminante dans l'enchaînement des faits. Le 28 février 1995, un groupe de quatre ou cinq hommes enlève à Tokyo un notaire de soixante-huit ans, Kariya Kiyoshi. Assez rapidement, la police dirige ses soupçons vers Aum Shinrikyô et lance un avis de recherche national contre un de ses disciples, Matsumoto Tsuyoshi, âgé de vingt-neuf ans.

19 Selon une rumeur persistante circulant au sein de la police et des médias, et dont les fondements sont difficiles à vérifier, cette affaire aurait déclenché l'attentat du métro. Aum Shinrikyô, par l'intermédiaire de disciples membres de la police, aurait été averti d'un raid imminent contre les locaux de la secte dans le cadre des recherches du notaire disparu. Coupable de l'enlèvement et de la mort de Kariya, la secte aurait lancé l'opération du 20 mars, afin de détourner l'attention de la police. Elle aurait aussi fait disparaître des pièces importantes permettant d'établir sa culpabilité dans plusieurs affaires. Bien des éléments d'un puzzle complexe se mettent en place donnant ainsi une relative crédibilité à la rumeur. Il se dira de plus en plus ouvertement, par exemple à la Cour de Tokyo lors des procès, que la secte, ayant été informée de l'imminence d'un raid policier à Kamikuishiki, notamment dans le cadre de l'affaire Kariya, aurait, avec l'attentat de Tokyo, voulu faire diversion.

20 Tokyo

21 Dans la nuit du 19 mars 1995, une bombe est lancée contre l'immeuble qu'occupe Aum Shinrikyô à Tokyo. Ce même jour, la police arrête trois membres d'Aum Shinrikyô relevant de la branche d'Osaka, soupçonnés d'avoir enlevé un étudiant qui tentait de s'échapper des locaux de la secte.

Le 20 mars 1995, du sarin se diffuse dans cinq wagons de métro sur les lignes Hibiya, Marunouchi et Chiyoda. Les premiers malaises sont constatés à $8 \mathrm{~h} 14$ le matin. La 
station Kasumigaseki est la plus touchée : les trois lignes de métro se croisent à cet endroit. Le lieu de l'attentat incarne la haute bureaucratie japonaise puisque la station dessert les Ministères des Affaires Etrangères, de l'Intérieur, de la Construction, des Transports, de l'Agriculture, du Travail, des Postes et Télécommunications, des Finances, la Cour du district de Tokyo et la Haute Cour de Tokyo et l'Agence de la Police Nationale. A peine plus loin, à trois ou quatre cents mètres au nord se trouvent encore les bâtiments de la police de la Ville de Tokyo et ceux du Ministère de la Justice, et au sud le Ministère de l'Education. Les fonctionnaires rejoignent leurs administrations généralement à $8 \mathrm{~h} 30$.

Très vite, arrivent sur les lieux le personnel du métro, la police, les pompiers, des équipes de secours d'urgence, la presse. Tous interviennent comme ils le peuvent tout au long de la matinée. L'organisation des secours s'improvise; la coordination laisse probablement à désirer, chaque spécialité s'en référant à son autorité de tutelle. Le gouverneur de Tokyo, Suzuki Shun'ichi, ne demande l'aide des spécialistes des Forces d'Auto-Défense ${ }^{4}$ qu'à $12 \mathrm{~h} 50$ et les équipes, accompagnées d'un certain nombre de militaires, ne seront sur place qu'à $13 \mathrm{~h} 30.3230$ personnes sont immédiatement hospitalisées dans cent soixante-neuf hôpitaux. Dans les heures qui suivent, le nombre de victimes ayant besoin de traitement augmente jusqu'à 5510 . Plus de 3800 personnes feront une déclaration à leur compagnie d'assurance. On trouve des victimes dans une vingtaine de stations de métro ${ }^{5}$. Sans doute, des erreurs importantes sont commises et c'est par la télévision que les équipes de secours intervenant sur place apprennent la nature du gaz toxique. Le sarin, développé en 1938 par les nazis pendant la Seconde Guerre mondiale, n'avait jamais été utilisé. Il se présente sous une forme liquide et dégage des vapeurs fortement toxiques dès son exposition à l'air. Dosé à $100 \mathrm{mg}$ par $\mathrm{cm} 3$, ce gaz, dès qu'il est respiré pendant une minute, provoque des troubles respiratoires graves, qui peuvent entraîner la paralysie et la mort.

Très vite, les médias parlent de la secte Aum Shinrikyô comme coupable possible. Alors que la population japonaise est encore très choquée, plusieurs autres faits se produisent semblant, dans un premier temps pourtant, disculper la secte. Début avril, le chef de la police nationale, Kunimatsu Takaji, est sérieusement blessé par balle. Surtout, le 19 avril, alors que de nombreux disciples de Aum Shinrikyô ont été arrêtés, des substances toxiques sont diffusées en différents points de la gare de Yokohama: environ cinq cents personnes doivent recevoir des traitements dans les hôpitaux.

Action policière et découvertes

Le 22 mars, sous une couverture médiatique exceptionnelle, et en relation avec l'enlèvement du notaire Kariya, 2500 policiers assiègent le complexe religieux de Kamikuishiki, où Aum Shinrikyô a implanté ses principaux bâtiments. Ils trouvent alors une cinquantaine de personnes, plus ou moins inconscientes, souffrant apparemment de malnutrition ou d'injection de drogues. Six d'entre elles sont dans le coma. La police confisque deux tonnes de chloroforme et d'éthane, quinze bouteilles d'éthylène, dix bouteilles d'un liquide jaune non identifié et des masques à gaz. L'ensemble de ces produits de base auraient pu permettre la production de 5,6 tonnes de $\operatorname{sarin}^{6}$, soit de quoi éliminer dix millions de personnes. Elle saisit également du matériel utilisable pour la fabrication de gaz et des quantités importantes de produits de base entrant dans la composition de la dynamite. Les policiers trouvent encore dans le coffre du gourou, Asahara Shôkô, au sein des bâtiments, sept cents millions de yens en espèces et 
dix kilos d'or en lingots. La secte a-t-elle pu être informée plusieurs heures auparavant de l'imminence d'un raid policier au sein de son quartier général de Kamikuishiki ${ }^{7}$ ?

Les pompiers inspectent l'héliport de la secte où se trouve un hélicoptère Mil Mi-17, de fabrication soviétique, et où sont entreposés sans autorisation ${ }^{8}$ plus de deux mille litres de fuel. L'appareil est la propriété de l'entreprise Maha-Posya à Tokyo, filiale de la secte dont le président est Asahara Shôkô. Aum Shinrikyô aurait acquis ce Mi-17 auprès d'une société de commerce autrichienne qui elle-même se le serait procuré par l'intermédiaire d'une société d'exportation russe.

Les policiers retournent sur place le 26 mars, dûment équipés de protections contre d'éventuelles émanations toxiques et accompagnés de canaris de détection. Ils perquisitionnent également différents bâtiments occupés par la secte. Ils découvrent des os humains dans l'un d'entre eux, à Fujinomiya, préfecture de Shizuoka.

Puis ils continuent leurs recherches dans d'autres installations de la secte. Le 14 avril, une perquisition a lieu simultanément dans cent vingt bâtiments occupés par la secte dans le pays. A Tomizawa, dans la préfecture de Gunma, ils découvrent une dizaine de machines-outils utilisables pour fabriquer des pièces de métal. Elles proviendraient de l'entreprise déclarée en faillite d'un des disciples. Dans une voiture, sont trouvées des pièces de fusils automatiques, d'un modèle russe.

Mi-mai, environ deux cents disciples ont été arrêtés. Parmi eux, quelques personnages clés de la secte, comme Hayakawa Kiyohide et vingt ingénieurs. Mais Asahara Shôkô reste introuvable et la police lance un avis de recherche national. Presque deux mois se sont écoulés depuis l'attentat du métro. C'est le temps qu'il aura fallu à la police pour établir avec certitude la culpabilité de Aum Shinrikyô dans l'attentat à travers les témoignages des disciples arrêtés et l'étude des documents saisis. Le 16 mai, retrouvé dans une cachette aménagée dans un bâtiment de Kamikuishiki, le gourou est arrêté.

31 Fin juillet, à Fujinomiya, enterrées dans les locaux de la secte, on trouve encore environ soixante-dix pièces métalliques, appartenant probablement à des missiles et à des bombes.

Juste un an après l'attentat de Tokyo, la police annonce que 613 mandats d'arrêt ont été présentés aux disciples d'Aum Shinrikyô et que 428 sont toujours en état d'arrestation.

33 La presse et l'opinion publique pendant de longues semaines s'étonnent d'apprendre que de nombreux disciples sont arrêtés pour des charges mineures, par exemple pour avoir conduit avec un faux permis ou pour s'être garé illégalement sur un parking privé. Les informations sont diffusées petit à petit, laissant s'établir des rumeurs et s'installer l'angoisse: la voiture mal garée contenait en fait des pièces de fusils automatiques et le chauffeur sans permis avait conduit un camion contenant du sarin. Diffusant au compte-gouttes l'information dont elle est seule détentrice, cachant l'arrestation de suspects ou leur mise en garde à vue, la police est critiquée, parfois par des unités concurrentes, pour sa stratégie d'information. Mais, graduellement est échafaudée la culpabilité d'un mouvement religieux.

La religion au Japon

Quelle est la place d'Aum Shinrikyô dans le contexte religieux japonais?

La pratique religieuse japonaise est multiple: il est fréquent que, pour une même personne, le mariage ait lieu selon des rites chrétiens, que les funérailles soient bouddhistes et que la vie soit jalonnée par l'observance de rites shintôistes. Ces deux dernières familles religieuses sont de loin les plus importantes au Japon. La foi est 
d'une manière générale peu profondément ancrée et, dans les zones urbaines, le respect des rituels est plutôt une affaire de convenances sociales.

Les grandes religions traditionnelles

Le Ministère de l'Education publie régulièrement des données qui donnent un aperçu de l'importance relative des grandes religions. Selon les derniers chiffres disponibles, le shintôisme, religion des kami (divinités tutélaires) attirerait 70,3 millions de fidèles. Cette religion est la plus ancienne au Japon. Elle vénère notamment la déesse du soleil, Amaterasu, considérée comme la génitrice de la dynastie impériale. Le bouddhisme a été introduit au Japon vers la moitié du VIe siècle par l'intermédiaire de la Corée. Il s'agit donc $\mathrm{du}$ bouddhisme $\mathrm{du}$ Grand Véhicule qui caractérise cette religion essentiellement en Chine, en Corée et au Japon. Il rassemblerait 81,3 millions de croyants. Ces deux religions sont divisées en différentes écoles qu'il convient d'appeler des sectes car ces écoles reposent sur des dogmes qui dévient de la doctrine d'origine. La secte Zen, par exemple, est fondée au XIIe siècle. Ces divisions ont eu lieu très tôt et ont perduré jusqu'à aujourd'hui, sans qu'il y ait de véritables renouvellements de ces dogmes et de leurs pratiques.

Le christianisme a fait son apparition au Japon au XVe siècle, mais n'a connu une expansion réelle qu'après la Seconde Guerre mondiale. Aujourd'hui, il y aurait 0,7 million de chrétiens au Japon.

A ces trois grandes religions s'ajoute une multitude de religions fondées sur des dogmes très variés et non reconnus par les religions mères et non classées comme shintôistes, bouddhistes ou chrétiennes par les autorités. Le nombre de croyants concerné est de 8,2 millions.

41 Le nombre total de fidèles s'élève ainsi à 160,6 millions, alors que la population japonaise est de 125 millions d'habitants, ce qui témoigne du caractère multiple des pratiques.

Les nouvelles sectes

Malgré les divisions en sectes, les deux grandes religions se côtoient et se superposent sans trop de difficulté. C'est essentiellement au XIXe siècle qu'apparaît une nouvelle génération de sectes. La plupart des nouvelles sectes japonaises se sont constituées sous la domination du shintôïsme d'Etat, ce qui les distingue des sectes occidentales. Un certain nombre d'entre elles entrent dans la spiritualité du shintôisme ${ }^{9}$, d'autres dans la sphère bouddhiste. Parmi ces dernières, plusieurs ont été fondées au moment de la Restauration de Meiji de 1868.

Un groupe de treize " nouvelles sectes " shintôistes vont devenir très importantes. Elles sont pour la plupart fondées au début du XIXe siècle. Par exemple, Kurozumikyô est instaurée en 1814 et obtient son statut de secte shintô indépendante en 1876. Avec un peu plus de quatre cent mille fidèles, elle est un des mouvements indépendants les plus importants du shintôisme. Tenrikyô, qui a souhaité en 1970 ne plus être classée parmi les sectes shintôistes, a été établie en 1838 et obtint sa reconnaissance officielle comme mouvement religieux en 1908. Cette secte compte aujourd'hui deux millions de fidèles. Les croyances de Tenrikyô incluent l'espoir d'une ère nouvelle, parfaite, qui se manifestera en tout premier dans le lieu sacré défini par la secte. Konkokyô existe depuis 1859 bien qu'elle ait été réellement organisée à partir de 1885 et qu'elle ait obtenu son statut d'organisation religieuse en 1900. Affiliée au shintôïsme et reposant 
sur une doctrine messianique, elle compte aujourd'hui 515000 fidèles sur l'ensemble du territoire national.

Tout le XIXe siècle est en mouvement du point de vue religieux. Avec la Restauration de Meiji, les deux grandes religions sont séparées, tandis que l'Etat renforce le shintôisme, tout en s'appuyant parallèlement sur la philosophie du confucianisme. Sur la fin du siècle et le début du XXe, le mouvement se poursuit avec, par exemple, Ômotokyô, établie en 1892, avant la guerre sino-japonaise (1894-1895), qui connut un remarquable développement, comme Tenrikyô et Konkôkyô. Hito no michi, secte relevant du syncrétisme shintô et fondée en 1930, promet la prospérité des affaires et l'harmonie au sein de la famille, et connaît une expansion rapide auprès des classes moyennes des villes et parmi les entrepreneurs.

Hommon butsuryû-kô, fondée en 1857, est d'essence bouddhiste. Et l'on peut encore classer parmi les " nouvelles sectes ", la très importante Soka Gakkai. D'affiliation bouddhiste, elle fut créée en 1930 et compte aujourd'hui 16,2 millions de fidèles. A partir de 1937, elle se développa en tant que nouvelle religion. La Risshôkoseikai, elle aussi d'affiliation bouddhiste, fut fondée à la même époque, en 1938, et revendique 6,5 millions de fidèles.

Toutes ces religions, dont seuls quelques exemples sont mentionnés ici, furent très contrôlées par le pouvoir qui intervenait pour qu'elles observent les " bons " rites shintô.

Parmi les sectes de cette génération, que l'on appelle donc les " nouvelles sectes " [shin shûkyô] dans le contexte religieux japonais, plusieurs affrontèrent une expérience dont le cas de Aum Shinrikyô va réactiver la mémoire. Hommon butsuryû-kô a été inquiétée de diverses manières au moment de la Restauration de Meiji, puis gagna une influence nationale à la fin du XIXe siècle. Mais, surtout, pendant la montée du militarisme japonais, plusieurs mouvements religieux furent persécutés. " Ômoto-kyô promet la venue du Miroku-no-yo (le monde idéal du bodhisattva Maitreya) en faisant revivre la vie agraire classique. Les enseignements de Ômoto-kyô reflètent les injustices sociales que crée le capitalisme. Les croyances s'étendent rapidement parmi les paysans, les citadins, les militaires et même auprès des intellectuels "10. De telles croyances n'étaient pas pour plaire au shintôisme d'Etat et au culte de l'empereur si bien que la secte commença à subir diverses pressions dans les années vingt. En 1935, elle fut accusée de crime de lèse-majesté et de violation de la Loi sur le maintien de l'ordre public. Ses activités furent complètement interdites jusqu'à la fin de la Deuxième Guerre mondiale. Hito no michi connut le même sort et fut dissoute en 1939. La Soka Gakkai, considérée comme portant atteinte au shintôïsme d'Etat, fut interdite en 1943, puis réactivée par l'un de ses deux fondateurs, Toda Jôsei, à partir de 1946. Elle nie et rejette toutes les autres religions qu'elle considère comme hérétiques. Toda Jôsei oriente le mouvement vers la politique dès 1955 et la secte connaît un nouveau développement à partir de 1964, à travers le parti Komeitô. Ses adhérents appartiennent majoritairement au monde des petites et moyennes entreprises et relèvent des salariés, des couches urbaines à revenu modeste et des femmes.

Murakami Shigeyoshi, dans l'article précité, rappelle que la plupart de ces nouvelles sectes restaient en relation étroite avec le mode de vie qui avait prévalu dans la sphère rurale. La communauté de croyances et de pratiques avait élaboré un esprit de corps. Et le recours à la magie et au chamanisme attiraient de nombreux adeptes. " Omoto-kyô, Hommichi, Hito-no-michi et Sôka-kyôiku-gakkai, toutes ces sectes diffusaient des 
enseignements religieux qui s'écartaient de ceux du shintôïsme d'Etat et furent accusées de lèse-majesté et de violation de la Loi sur le maintien de l'ordre public. Toutes furent sévèrement opprimées "11.

Après la Deuxième Guerre mondiale, ces sectes purent revivre. Elles changèrent souvent de nom et continuèrent leur développement. La liberté de croyances et la séparation de l'Eglise et de l'Etat furent établies par l'article 20 de la Constitution qui prend effet en 1947.

51 " La liberté de religion est garantie à tous. Aucune organisation religieuse ne recevra de privilège de la part de l'Etat ou ne se verra imposer une quelconque autorité politique ; aucun individu ne sera obligé de participer à des actes, célébrations, rites ou pratiques d'ordre religieux; l'Etat et ses organes n'interviendront pas au sein de l'éducation religieuse ou de toute autre activité religieuse ".

52 Mais la persécution dont elles ont été l'objet pendant la période de montée du fascisme japonais reste dans toutes les mémoires et tout le débat sur la dissolution de la secte Aum Shinrikyô s'inscrit dans cette histoire-là. Aujourd'hui, il y a environ sept cents nouvelles sectes au Japon.

Les nouvelles nouvelles sectes

54 Les années quatre-vingt voient arriver de nouveaux mouvements religieux et cette dernière vague de création que connaît le Japon ressemble en bien des points à ce qu'ont connu les Etats-Unis et l'Europe à la même époque. Ces mouvements récents furent désignés sous l'expression " Nouvelles nouvelles religions " [shin shin shûkyô]. Se présentant comme différents et des religions traditionnelles et des Nouvelles sectes, ils sont aussi des sectes.

Les nouveaux mouvements religieux occidentaux et japonais présentent divers points communs, outre le moment de leur apparition. Bien souvent, ils connaissent une extension rapide, sans doute parce qu'ils mettent l'accent sur le recrutement, fixant parfois des objectifs concrets aux recruteurs. Le fondateur ou gourou est toujours une personne vivante, à l'origine ordinaire. Les dogmes sur lesquels reposent ces mouvements intègrent des éléments des religions traditionnelles, les réinterprètent et leur donnent des prolongements nouveaux. Presque toujours, les pratiques magiques tiennent une grande place, qu'il s'agisse de redonner le bonheur, la santé ou de délivrer les croyants de leur mal-être. Surtout, le centre de la spiritualité est la personne ellemême. Tous ces mouvements fabriquent un sentiment d'appartenance à la communauté religieuse, ce qui constitue une de leurs principales forces.

$56 \mathrm{Au}$ Japon, les nouvelles nouvelles sectes se posent très clairement contre les religions établies et elles ont, légalement, une existence et un statut indépendant par rapport aux sectes nouvelles ou traditionnelles. C'est à cette génération qu'il faut rattacher Aum Shinrikyô. En réalité, les Nouvelles nouvelles sectes sont apparues au Japon après la Deuxième Guerre mondiale, mais se sont multipliées surtout pendant les années quatre-vingt. Elles intègrent presque toujours la science moderne. Elles savent utiliser la musique, organiser de grands spectacles. Leurs grands meetings de masse peuvent leur donner d'un seul coup une grande notoriété. Par exemple, Aum Shinrikyô tenait des meetings fréquents consacrés à Ozaki Yukata, chanteur très populaire disparu de manière mystérieuse en avril $1992^{12}$. La secte attirait ainsi les nombreux fans qui espéraient entendre des nouvelles de leur idole perdue et tentait de les séduire en vue d'un recrutement. 

disparaît, le mouvement peut s'effondrer car il agit comme un leader charismatique. De plus, il est le chef spirituel : il signe les textes de référence, dicte le dogme et est seul habilité à le modifier, il définit les rites et indique leur sens. Il a un rôle social important : il diffuse l'image du mouvement à l'extérieur, en participant à des débats ou en représentant le mouvement vis-à-vis de ses interlocuteurs. Il a également un rôle organisationnel capital : il est celui qui dirige l'organisation religieuse et décide de sa forme. Aum Shinrikyô répond à toutes ces caractéristiques. lui donne une densité tout à fait exceptionnelle. Si bien que les données officielles font état, pour l'année 1994, tous types de sectes confondus, de 231428 organisations différentes ${ }^{13}$. Selon l'Agence pour les Affaires Culturelles qui, au sein de Ministère de l'Education est directement en charge de ces questions, une centaine de groupes religieux demandent leur reconnaissance chaque année. Parmi ceux-ci, environ soixante procèdent à leur auto-dissolution, presque toujours parce qu'ils n'arrivent pas à se maintenir en tant qu'organisation au cours de la première année. Ceux qui demandent leur reconnaissance doivent apporter la preuve qu'ils constituent une organisation religieuse dont le seul but est de dispenser leur doctrine, quelle qu'elle soit; ils doivent pratiquer des rites et diffuser leur enseignement à leurs fidèles dans leurs locaux.

En japonais, on parle donc de shûkyô, de shin-shûkyô et de shin-shin-shûkyô, respectivement religions, nouvelles religions et nouvelles nouvelles religions ou sectes, mais on parle également de karuto, notamment pour des mouvements comme Aum Shinrikyô. Karuto est la prononciation japonaise de l'anglais cult et est assorti des mêmes nuances péjoratives que le terme français " secte ".

Aum Shinrikyô

Aum Shinrikyô est dans tous les sens du terme une secte, car cette organisation revendique sa parenté spirituelle avec le bouddhisme tout en critiquant, parfois fortement, les autres sectes de cette religion alors que le bouddhisme considère les croyances et les pratiques de Aum Shinrikyô comme déviantes.

Dès l'attaque au sarin de Tokyo, le nom de la secte circule bien que les preuves pour inculper ses membres manquent. Mais quelle est cette secte? En fait, bien peu d'informations existent alors: les universitaires spécialistes des nouvelles religions n'ont apparemment pas étudié ce mouvement particulier ou, en tout cas, ils n'ont rien publié. Les médias ne se sont intéressés à Aum Shinrikyô que tout à fait exceptionnellement. Le Sunday Mainichi avait par exemple consacré une série d'articles à la secte en 1989 , secte contre laquelle commençaient à se formuler des plaintes émanant soit de son voisinage, soit de familles de jeunes disciples. Le journal dénonçait le caractère antisocial de la secte. Finalement, les quelques rares personnes qui détiennent au moment de l'attentat des informations sérieuses sur la secte sont un cabinet d'avocats de Yokohama d'où un jeune avocat, Sakamoto, a disparu en 1989, ainsi que les personnes et associations qui, dans l'ombre, cherchent à soulager des familles de disciples de Aum Shinrikyô ou d'autres sectes. Comme les ex-disciples resteront longtemps silencieux, la plupart des informations qui circulent après l'attentat sont émises et contrôlées par la police.

Cultures \& Conflits, 29-30 | automne-hiver 1998 

ouvre uneé cole de yoga dans un des plus grands centres commerciaux de la capitale, à Shibuya. Il enseigne à ses élèves diverses techniques de méditation. Il lui faut à peine deux ans pour poser les fondations de son propre mouvement, qu'il crée en 1986 : Aum Shinrikyô Shinsen no Kai ${ }^{16}$. Il prend le nom religieux de Asahara Shôkô, évoque alors ses premières expériences de lévitation et affirme avoir reçu la mission divine de construire sur terre le royaume de Shambhala. Il publie très vite Chônôryoku himitsu no kaihatsuhô [Comment développer vos pouvoirs extraordinaires secrets]. Il est alors entouré d'une quinzaine de fidèles, pour la plupart élèves de son école de yoga. A partir de là, il s'adonne à une activité éditoriale intense, se déplace de plus en plus souvent. Un gourou est né. Sa mission le conduira, avec son mouvement, aux événements de 1994 et de 1995.

L'année suivante, en 1987, Asahara Shôkô rencontre le Dalaï-lama en février et se rend en Inde en juillet où, dit-il, il reçoit l'ultime délivrance dans la chaîne de l'Himalaya. Au cours de ce mois, il change alors le nom de son mouvement religieux en Aum Shinrikyô [Religion de la Vérité Suprême], décide de désormais se consacrer entièrement à la secte et développe très rapidement ses activités ${ }^{17}$. L'objectif religieux consiste à se débarrasser de ses mauvais karma pour la vie présente et pour les vies futures en s'infligeant des souffrances psychiques et physiques. Cette fin justifie tous les traitements que subissent les disciples. De nombreuses pratiques internes à la secte relèvent du yoga et les étapes d'un entraînement très poussé se succèdent dans un enchaînement sophistiqué et défini par Asahara Shôkô. A cela s'ajoutent de longues périodes de prostration et l'ensemble doit conduire ceux qui observent cet enseignement vers le gedatsu, le stade suprême de l'Illumination, que seul le gourou a alors atteint.

69 Sans nul doute, Asahara Shôkô est à ce moment-là un grand leader. Il apparaît comme tel en 1985. Des photographies le montrant en lévitation impressionnent alors 
beaucoup de ceux qui l'approchent. Jusqu'à la fin, son charisme ne le quittera pas. Tous les récits des ex-disciples en témoignent. Mais aussi, de nombreux Japonais qui n'ont jamais eu de contact direct avec Aum Shinrikyô, se souviennent simplement de l'impression que Asahara Shôkô donnait lors de ses passages télévisés : un calme, un esprit logique, une voix douce et bienveillante, une personnalité qui séduisait naturellement et souvent intellectuellement ceux qui croisaient son chemin. "J'admire le maître suprême parce qu'il nous dit ce que personne d'autre ne pourrait expliquer aussi clairement et aussi logiquement ", dit un disciple, étudiant en doctorat, dont la foi n'est pas ébranlée ${ }^{18}$. Des religieux d'obédiences diverses m'avouent aussi, hors micro, l'intérêt qu'il suscitait, y compris auprès de ses plus farouches détracteurs. En effet, sa capacité à contredire sur le fond religieux l'immobilisme des Eglises traditionnelles séduisait de nombreux jeunes dignitaires religieux qui attendaient depuis si longtemps une critique qui appellerait un changement qu'eux-mêmes n'osaient initier tant les hiérarchies sont strictes, pour ne pas dire, souvent, intolérantes.

En août 1989, Aum Shinrikyô obtient sa reconnaissance comme organisation religieuse auprès de la Ville de Tokyo. Le mouvement reçoit donc de fait tous les privilèges de son nouveau statut, notamment l'exonération fiscale pour ses revenus sous forme de dons, et un allégement substantiel pour les autres types de revenus. La secte a d'ores et déjà des représentations dans presque toutes les grandes villes. Elle installe son siège à Tokyo et son centre de formation à Fujinomiya, dans la préfecture de Shizuoka, peu éloignée de la capitale.

71 Recrutement, croyances et rites

72 Comme dans la plupart des mouvements religieux, Aum Shinrikyô a deux types de disciples : les moines, hommes et femmes qui vivent en communauté, ou shukke [soit, mot à mot, ceux qui ont quitté la maison] et les adeptes laïcs qui continuent à vivre dans la société, les zaike [ceux qui sont présents à la maison]. Dans le cas de Aum Shinrikyô, la communauté des shukke est fermée; seuls quelques-uns gardent des contacts avec l'extérieur pour les besoins de la secte. Les shukke reçoivent chacun un nom religieux qu'ils utilisent pour se désigner, une fois entrés au sein de la communauté. Au printemps 1995, au moment de l'attentat du métro de Tokyo, la secte fait elle-même état de 1115 shukke et de dix mille disciples au Japon. D'après certains témoignages d'anciens shukke, les chiffres réels seraient plus élevés d'environ $50 \%$. Comparée à d'autres sectes japonaises de la même génération, l'expansion de Aum Shinrikyô, quoique rapide, n'a pas été des plus spectaculaires. Les shukke de ce mouvement religieux présentent certaines caractéristiques : $62,2 \%$ ont moins de trente ans, 90,2 \% moins de quarante ans; la plupart ont des diplômes de niveau élevé. Les recrutements ont visé tout particulièrement les élites, ce qui, là encore, n'est pas propre à Aum Shinrikyô, avec des méthodes qui passaient pour très agressives, notamment parce que des dons importants étaient exigés.

La dimension eschatologique des croyances, bien que présente dès le début, n'apparaît pas comme centrale au début et les premiers textes publiés par Asahara Shôkô insistent plus sur les relations avec le bouddhisme et les références aux religions tibétaines. L'ensemble s'appuie sur la pratique du yoga, présentée comme la voie pour atteindre l'Illumination. Les phénomènes paranormaux ne sont pas absents au sein de la secte ${ }^{19}$. Dans un premier temps, Asahara Shôkô fait état de la caution que lui apporterait le Dalaï-Lama. Une photographie où les deux hommes se tiennent côte à côte est largement diffusée au sein de la secte. La Chine utilisera d'ailleurs cette relation 
éphémère pour servir sa propre cause, faisant courir le bruit par des canaux officieux à Taiwan que le Dalaï-Lama chercherait à réformer le bouddhisme japonais en se servant de Asahara Shôkô. Par la suite, notamment après mars 1995, le Dalaï-Lama marquera clairement ses distances. La secte se déclare elle-même comme bouddhiste, référence religieuse centrale pendant cette première période. Seul Asahara Shôkô définit le dogme et est habilité à le faire évoluer. Il a seul le pouvoir de déclarer la progression des disciples au sein de la hiérarchie religieuse et d' "illuminer " les plus fidèles. " Seuls ceux qui auront pratiqué, ceux qui auront été choisis dans ce monde, seront élevés à un monde supérieur par leur gourou " ${ }^{20}$. Il décide donc qui a atteint l'immortalité, qui est condamné. Cette caractéristique se retrouve dans d'autres mouvements religieux fondamentalistes.

74 La fidélité au dogme qui passe par la soumission au gourou autant que la régularité des pratiques conduisent vers le gedatsu, cet état de délivrance ultime qui est détachement complet, physique et mental, de tous les liens avec le monde réel, qu'il s'agisse de la consommation, du sexe ou des sentiments. D'une certaine façon, le dogme est une critique de la modernité, d'une rationalisation et d'une individualisation de plus en plus perceptibles. Asahara Shôkô répète que le monde réel n'est qu'une illusion. Et il cherche à établir une " morale " fondatrice, pivot des croyances de la secte, la morale des " quatre vertus infinies ": l'amour, la compassion, le bonheur et l'équanimité (égalité d'âme) [envers tout et tous] sont une et indivisibles: " Supposez que vous marchez dans la rue. Et vous voyez qu'une ambulance emmène quelqu'un sur une civière. Vous accourez pour vous assurer qu'il ne s'agit pas de votre père ou de votre mère. Comme vous ne connaissez pas le blessé, que faites-vous? Vous reprenez votre marche? Cela ne vous concerne pas? Mais réfléchissez un moment. Cette personne n'est pas votre parent, mais peut-être l'était-elle dans une vie antérieure? Dans le passé, n'avons-nous pas eu un nombre infini de vies, sans commencement ? N'est-il pas possible alors que chaque chose ait été notre père ou notre mère à un moment ou à un autre ? Ne devons-nous donc pas considérer et aimer chaque chose comme notre père ou notre mère? Une fois que l'on a réalisé cela, on peut pleurer la souffrance de tous (...) C'est ce qui fonde l'esprit d'égalité : aimer également toutes les créatures, éprouver une compassion égale pour tout "21.

75 Atteindre le gedatsu signifie parvenir à la liberté et au bonheur absolus. Bien que seul Asahara Shôkô connaisse l'état de gedatsu, il s'engage vis-à-vis de ses fidèles à conduire le plus grand nombre possible, le plus vite possible, vers le gedatsu, d'où la nécessité de se livrer à des enchaînements relativement complexes de pratiques qui s'appuient sur un entraînement physique, psychologique, mental et spirituel où le yoga tient une place centrale. Les différentes phases et étapes doivent se succéder au cours d'un enchaînement long et précis suivant un programme préétabli et dicté par le gourou. Asahara Shôkô passe pour un expert dans la pratique du yoga. Et c'est dans son centre de yoga qu'il a recruté ses fidèles de la première heure, ceux qui le suivront jusqu'au bout. C'est donc d'abord sur le yoga qu'il fonde les pratiques. La méditation occupe une place très importante et la lévitation est un des buts des pratiques. Une surabondance de cérémonies et de rites, pour lesquels l'imposition des mains joue un grand rôle, et devant être accomplis dans un ordre bien précis, complète ce parcours spirituel censé conduire l'adepte sur le chemin de la Vérité.

76 L'ensemble du discours religieux, dès le début, met l'accent sur la mort et sur la vie après la mort. En réalité, on ne prononce pas le terme de mort au sein de la secte qui 
désigne ce moment par des termes d'origine sanskrite. On parle ainsi volontiers de poa, tout comme dans d'autres sectes, au sein de l'Ordre du Temple Solaire par exemple, on parle de " grand voyage " ou de " transit ".

77 Sans doute beaucoup plus ouvertement que ne l'avait fait aucun autre leader religieux avant lui, Asahara Shôkô mène une critique systématique et violente envers d'autres mouvements religieux. Plusieurs sectes deviennent la cible privilégiée de Aum Shinrikyô. Il donne l'impression de soulever les côtés poussiéreux des religions traditionnelles et nouvelles et sa présence même rend possible un réel débat non seulement entre sectes, mais entre divers pans de la société et le monde religieux. Mais les discussions qu'il a ouvertes ne sont pas seulement des débats de fond concernant la religion. De réels conflits opposent Aum Shinrikyô à d'autres sectes. Notamment la Soka Gakkai que Aum Shinrikyô désigne comme coupable dans l'affaire de la disparition de Kariya. A l'origine, les dirigeants de Aum Shinrikyô eurent l'idée de fabriquer du sarin probablement pour éliminer Ikeda Daisaku, président d'honneur de la Soka Gakkai. A la mi-octobre 1993, Asahara Shôkô aurait ordonné à certains de ses disciples de tuer des personnalités religieuses, mais les opérations n'ont pas réussi. Après l'attentat de Tokyo, la Soka Gakkai évoque publiquement la possible culpabilité de Aum Shinrikyô qui l'accuse en retour de diffamation.

Jusqu'en 1989, hormis les articles du Sunday Mainichi, Aum Shinrikyô autant que Asahara Shôkô sont sans histoire. Ceux qui pourraient avoir des informations agissent sur le terrain de manière anonyme. Certes, les shukke se retirent du monde, mais comme le font les moines et prêtres dans de nombreuses religions. Ils abandonnent tous leurs liens avec l'extérieur, ils font don de leurs biens à la secte en renonçant à tout attachement au monde réel. Bien que cette coupure radicale avec le monde réel paraisse incompréhensible à certaines familles qui ont ainsi perdu de vue un proche, on peut dire que durant cette première étape de la construction de la secte, dogme et pratiques religieuses et sociales restent à l'intérieur d'une certaine logique. La vie à l'intérieur du mouvement religieux avant 1989, et notamment au sein du groupe des shukke, est assez peu connue. Tout se passe comme si la secte à travers ses shukke se désocialisait, éliminant peu à peu les sources des rapports qu'elle entretient, individuellement et collectivement, avec le reste du monde. Mais de nombreuses religions imposent ce renoncement à leurs moines. Une séparation du social qui, somme toute, $\mathrm{n}$ 'a rien d'étrange.

Le tournant de 1989 : tentative de retour au social et apparition de la violence

Août 1989 marque l'entrée de Aum Shinrikyô dans une nouvelle phase. A cette date, le mouvement acquiert son statut d'organisation religieuse. Désormais officiellement et socialement reconnu, il bénéficie, de plus, d'avantages importants. Le gourou choisit ce moment pour tenter avec son organisation un retour vers le social. 


\section{NOTES}

1. Dans ce texte, tous les patronymes japonais sont écrits à la japonaise, le nom de famille précédant le prénom. A plusieurs reprises, des montants sont mentionnés en yens : sur la période dont il est question ici, 100 yens $=5 \mathrm{FF}(1000000$ yens $=50000 \mathrm{FF}$; 1000000000 yens $=50000$ 000FF).

2. En novembre 1985, sur le réseau de chemins de fer, les câbles de communication avaient été sectionnés et le système de signalisation endommagé en une trentaine de lieux répartis sur l'ensemble du territoire, au moment où une grève touchait les Chemins de fer nationaux ; en mai 1986, bien qu'il n'y eût pas de blessés, l'explosion de bombes fumigènes dans dix-neuf stations de train dans la région de Tokyo avait troublé le Sommet des pays industrialisés qui était réuni dans la capitale ; en juin 1990, des bombes à retardement posées par des groupes d'extrême-gauche protestant contre l'institution impériale, sont trouvées dans huit trains à Tokyo ; en avril 1993, des incendies sont allumés, probablement par des extrémistes de gauche, dans quatre temples de Tokyo pour protester contre la visite de l'empereur à Okinawa; en décembre 1992, une dizaine de voitures appartenant à l'aéroport de Narita sont détruites, fait attribué à des extrémistes de gauche.

3. Les informations contenues dans ce rapport proviennent de plusieurs sources, et notamment :

- la presse japonaise quotidienne (Asahi Shinbun, Japan Times, entre autres) et hebdomadaire ou à périodicité moins fréquente (Bungei shunju, Chûô kôron, Aera, voire Shokun, Takarajima...) ; les comptes-rendus d'audiences lors des procès des disciples ; les entretiens approfondis que j'ai menés en mars et en septembre 1996 avec une vingtaine de personnalités liées aux affaires de la secte (ex-disciples de Aum Shinrikyô, personnalités religieuses et politiques, avocats, psychiatres...) ; les discussions que j'ai pu avoir avec des universitaires japonais spécialistes. Toutes ces sources ont été systématiquement croisées pour l'analyse.

4. Le Japon qui, constitutionnellement, ne peut avoir d'armée, a un corps militaire, les Forces d'Auto-Défense, dans lesquelles on ne peut entrer comme militaire que sur une base volontaire.

5. Cf. annexe.

6. Il semble qu'il n'y ait pas de difficulté pour se procurer ce type de produits, bien que le nombre de fournisseurs au Japon soit réduit. L'acheteur doit fournir son identité, le nom de l'entreprise concernée par l'achat et celui de ses représentants. Il doit laisser un numéro de téléphone et préciser l'utilisation ultérieure du produit.

7. Notamment à travers un message lancé sur le serveur Nifty, qui compte près d'un million d'abonnés au Japon.

8. La réglementation exige une autorisation pour un stockage d'essence ou de fuel supérieur à 1200 litres.

9. Par exemple Jikkô-kyô ou Fusô-kyô, qui relèvent de ce que l'on appelle l'ascétisme de montagne, et qui vouent un culte au Mont Fuji, ou Ontake-kyô qui vénère le Mont Kiso. Il y a d'autres sectes shintô qui utilisent leur interprétation du dogme.

10. Cf. Murakami Shigeyoshi, "New Religions in Japan", East Asian Cultural Studies, March 1972, 11, 1-4, p. 20-21. 
11. Cf. Murakami Shigeyoshi, op. cit., p. 22-23.

12. Le chanteur avait été trouvé intoxiqué dans les rues de Tokyo et était mort peu après. Des pétitions ayant collecté en tout plus de cent mille signatures réclamaient une enquête et insinuaient qu'il s'agissait d'un homicide.

13. Source : Monbusho, 1995 Shûkyô nenkan. Ces chiffres correspondent au nombre d'organisations religieuses [shûkyô dantai] et désignent les temples, monastères, églises dont le but premier est la diffusion des enseignements religieux, la réalisation des cérémonies et fonctions religieuses et l'éducation des croyants, ainsi que les sectes [shûha], les fédérations [kyôha], les ordres [shôdôkai] et diocèses [shikyôku] et autres organisations qui comprennent les organisations religieuses précédemment désignées. Parmi ces organisations religieuses, 184000 sont enregistrées dans les préfectures et, au sein de ces dernières, 177000 sont des temples ou églises qui, bien qu'enregistrés en tant qu'organisations autonomes, sont souvent membres d'une organisation religieuse plus importante.

Parmi les organisations religieuses reconnues, 90699 sont d'affiliation shintôiste, 89 218 sont affiliées au bouddhisme, 9335 au christianisme, 42176 sont classées sous " autres " affiliations. A ces chiffres s'ajoutent encore 47023 organisations religieuses sans désignation.

14. Médecine orientale très couramment utilisée au Japon et qui consiste à traiter les maux en posant sur la peau, en des endroits précis, de petits cônes ou cylindres confectionnés à base de mélanges d'herbes que l'on fait se consumer directement sur la peau.

15. God Light Association, plus connue sous le nom de " GLA ", fait partie des sectes de la dernière génération qui ont connu un développement rapide. Ses croyances accordent beaucoup de poids aux vies antérieures dont les fidèles gardent la mémoire. Au nom de cette mémoire, ils seraient capables de parler le langage qu'ils parlaient au cours de leurs vies précédentes, si bien que les croyants actuels s'expriment " en langues".

16. Aum Shinrikyô est un terme sanskrit qui signifie création, permanence de la création, destruction de l'univers.

17. Les informations biographiques qui précèdent reposent largement sur les renseignements fournis par Ishii Kenji, Religion in Japanese Culture, Tokyo, Kodansha International, 1996.

18. Propos rapportés par The Japan Times, 1.6.1995.

19. Comme dans d'autres sectes de la même génération : Mahikari, GLA [God Light Association], Agonshû, Kôfuku no Kagaku, Worldmate.

20. Asahara Shôkô, Declaring myself the Christ, 1992, p. 131.

21. Asahara, 1987.

INDEX

Index géographique : Japon

Mots-clés : guérillas et organisations clandestines, religion, violence, attentat 